\title{
Acknowledgement to reviewers 2017
}

(C) Springer-Verlag GmbH Austria, part of Springer Nature 2018

We would like to thank the following reviewers who have taken part in the peer-reviewing process for ADHD Attention Deficit and Hyperactivity Disorders in 2017. Their efforts and advice essentially contributed to maintain the high quality of the journal.

Raman Baweja, United States Stephen Becker, United States Gregor Berger, Switzerland Kim Bernosky, United States Nóra Bunford, Hungary Nicholas Carleton, Canada Pieter-Jan Carpentier, Netherlands Arthur Caye, Brazil Andrew Coogan, Ireland Carsten Drepper, Germany Karin Egberts, Germany Jeffery Epstein, United States John Fayyad, Lebanon Stefanie Fekete, Germany Mark Freeston, United Kingdom Thomas Gift, United States David Goodman, United States Yvonne Groen, Netherlands Daniela Hartmann, Germany
Rebeca Heidbreder, United States Leona Hellwig, Germany Jamie Humphrey, United States Thomas Jans, Germany Maija Konstenius, Sweden Kerstin Krauel, Germany Klaus Lange, Germany Wolfgang Lenhard, Germany Michael Lensing, Norway Carlos Maia, Brazil Barrie Marchant, United States Ariel Martinez, United States Ivo Marx, Gemany Keith McBurnett, United States Anneke Meyer, South Africa Mónika Miklósi, Hungary John Mitchell, Australia Hemant Nandgaonkar, India Florence Phillipp-Wiegmann, Germany
Guilherme Polanczyk, Brazil Pradeep Rao, Australia Wolfgang Rauch, Germany Tilmann Reinelt, Germany Tobias Renner, Germany Janos Rethelyi, Hungary Luis Rohde, Brazil Roberto Sassi, United States Frank Sattler, Germany Megan Schaefer, United States Johanna Schmid, Germany Esther Sobanski, Germany Mary Solanto, United States Eric Taylor, United Kingdom Margaret Thompson, United Kingdom Jaisoorya Ts, India Clemens von Schacky, Germany Pal Zeiner, Norway 